\title{
Adapting and Delivering Care during the COVID-19 Pandemic: Staff Diary from the Mood Disorders Unit in Singapore
}

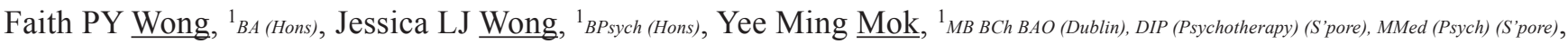
Nisha Chandwani, ${ }^{1}$ MBBS (Baroda), MRC (Psych) (UK)

The Mood Disorders Unit (MDU) is a specialised unit within the Institute of Mental Health (IMH), providing inpatient and outpatient services for major depression and bipolar affective disorder. The inpatient unit provides a therapeutic milieu with group therapy, case management and an active peer support programme. Upon discharge, patients are followed up by the same multidisciplinary MDU team, and have access to an outpatient group programme called Day Therapy Programme (DTP). To the best of our knowledge, the DTP is one of its kind available in Singapore that uses group dynamics as a primary therapeutic intervention. The aim is to promote recovery, independence and hope, in a respectful, mutually responsible and collaborative treatment environment.

The psychological impact of the COVID-19 pandemic on the general public, and on healthcare staff morale has been previously described. ${ }^{1,2}$ Here we describe the impact of COVID-19 measures on MDU staff and its patients.

On 23 January 2020, Singapore confirmed its first case of COVID-19. Further precautionary surveillance measures were effected, e.g. increased temperature screening, incoming visitor restrictions and quarantine measures. ${ }^{3}$ On 4 February 2020 , the first locally transmitted case was announced. The Disease Outbreak Response System Condition (DORSCON) ${ }^{4}$ was raised to Orange 3 days later, after 3 new cases of unknown origin emerged.

\section{Impact on Staff}

IMH implemented COVID-19 control measures to keep all staff and patients safe, while maintaining the provision of essential mental health services and resource management. ${ }^{5}$ All non-essential leave was cancelled to facilitate manpower planning. The sudden change and uncertainty affected staff morale, especially those with families overseas, as they had to manage the additional strain of staying away from their family and worrying for their family's safety. Staff were asked to wear surgical masks at all times and record their temperatures twice a day. There were restrictions on non-essential movements of staff and patients between healthcare institutions to reduce transmission risk. Centralised screening of travel history and temperature for all patients and visitors to IMH was mandatory upon entry. There were regular communications, town hall meetings and updates of the situation and measures in the hospital. At the unit level, regular assurances and check-ins were done between members of the MDU team.

Over the span of 3 days after DORSCON Orange, IMH incrementally implemented even stricter measures to aid in the national pandemic control effort. This had several implications on MDU's usual operations and delivery of patient care. MDU staff had to adopt splitteam arrangements that involved relocating of offices and limited face-to-face interaction, impacting regular case discussions essential in the management of patients. Family sessions and corroborative history taking from family and friends, which are usually done face-to-face and are vital in psychiatric clerking and formulation, were shifted to phone or video calls as far as possible, due to visitor restrictions. Discharge planning was affected, as the usual practice of home leave and home visits was restricted because of cross-contamination risk. Notwithstanding the challenges posed, staff adapted quickly by learning to use videoconferencing equipment to conduct its activities while working through confidentiality and technical concerns. Psychiatric nurses learnt to be more vigilant, as patients with respiratory symptoms had to be identified and isolated promptly.

As all staff were required to wear masks when meeting face to face with patients, patients reported difficulties in building rapport due to the inability to see a therapist's facial expressions. Staff also reported

\footnotetext{
${ }^{1}$ Mood Disorders Unit, Department of Mood and Anxiety, Institute of Mental Health, Singapore

Address for Correspondence: Ms Faith Wong Pih Yng, Department of Mood and Anxety, Institute of Mental Health, 10 Buangkok View, Buangkok Green Medical Park, Singapore 539747.

Email: faith_py_wong@imh.com.sg
} 
having breathing difficulties when projecting their voice to a group of patients during group therapy sessions or outdoor activities (sports and horticultural therapy), in Singapore's warm and humid climate.

The increase in local transmission and unlinked cases led to the announcement of an elevated set of safe distancing measures termed 'circuit breaker' on 3 April 2020. ${ }^{6}$ This resulted in a closure of workplaces, except for essential services. Many decisions, such as classifying psychological services as non-essential, evoked strong feelings among MDU staff and patients. An internal review of essential and non-essential services at the hospital and departmental level proved to be harder than expected. In staff, this was amplified by a sense of loneliness and anxiety, as working from home, eating alone at work, splitting of teams, holding online meetings and therapy sessions became a new routine to adjust to quickly. This was on top of existing personal commitments that staff had to manage, such as the care of children and elderly parents who were also home-bound.

\section{Impact on Inpatients}

Each inpatient was initially limited to 1 designated visitor. This was later tightened to no visitors at all during the circuit breaker period. As a result, warded patients faced a growing sense of isolation and uncertainty of their recovery. They could not connect with what was happening in the community, and there were fewer inpatient therapy groups for them to participate in due to the reduced manpower on-site. Empowering patients for recovery became a bigger challenge for the staff. To overcome this, videoconferencing-enabled laptops and smartphones were provided to patients to link up virtually with visitors and off-site staff. As they anticipated the struggle to cope with the pandemic and its coupled social isolation, alongside a reduction in face-to-face services available after discharge, some inpatients eventually preferred a longer hospitalisation time because they perceived the ward to be a safer and more conducive environment for recovery.

\section{Impact on Outpatients}

Outpatients faced multiple changes. While those requiring closer follow-up or depot injection continued to be seen at the clinic, non-urgent outpatient consultations were rescheduled, with the option of medication delivery where possible, to reduce visitor numbers and encourage safe distancing in the clinics. IMH started offering teleconsultations for outpatient appointments during the circuit breaker period, so that patients could consult with their doctors from home. Those who had presented at outpatient appointments with elevated temperatures were either seen by psychiatrists at the screening centre via videoconferencing, or experienced shorter therapy sessions after waiting for their temperatures to stabilise within an acceptable range. While there appeared to be no direct impact of the pandemic on patients' symptoms or relapse rates (e.g. no increased anxiety or ruminations on catching the virus), there was a noticeable impact of safe distancing measures on patient stress levels and coping mechanisms. As the outpatient DTP and majority of community outreach services were suspended, some patients reported a perceived loss of supportive networks and could rely only on an existing Telegram chat group for support. Other challenges encountered by them included difficulty in utilising their usual coping strategies involving outdoor activities, having to adapt to new daily routines, being in close proximity to stressors in the home environment, and having a change of psychiatrist. With combined efforts from the allied health professionals, frequent check-ins were made to provide additional support for outpatients, including normalising their feelings and reminders to practise self-care.

On the other hand, some patients were observed to have adjusted positively to the changes brought on by the pandemic. Elderly patients - specifically those who lived with their children - appeared to have benefitted from increased interaction with their children, who were once busy with their work or social life. Also, a large number of patients perceived the experience of working from home as an opportunity to be away from a stressful office environment. There was a noticeable decline in their work stress and anxiety levels.

\section{Bringing DTP Online}

As safe distancing appears to be the new norm in the foreseeable future, the MDU team decided to resume its DTP virtually via teleconferencing to provide continued support and a sense of connectedness for its outpatients. ${ }^{7}$ Our Peer Support Specialists, Medical Social Workers and Music Therapist collaborated to facilitate open groups every weekday, while our Art Therapist and Case Managers planned to resume structured and closed groups (Interpersonal and Social Rhythm Therapy, Art Therapy). There were apprehensions surrounding patients' perceived safety on a virtual platform and patients' accessibility of a safe physical 
environment during circuit breaker to participate in online groups. However, within 2 weeks, the team managed to address issues related to registration and consent, safety contract of online groups, and administration of rating scales.

The virtual DTP was initially rolled out to existing patients of the physical DTP, and subsequently new patients were referred to it. Younger patients were generally more open to the new virtual format of the groups, although a small number still preferred face-toface sessions. Numerous patients of the older generation declined to participate in the virtual group, citing unfamiliarity with technological platforms for support groups, despite their having an Internet access or a supportive family environment. There was an average satisfaction score of $86 \%$ from our Client Satisfaction Questionnaire. Feedback from participants suggested that the groups helped them feel less lonely, offered practical support and coping mechanisms, and offered a safe space to share how they were feeling.

As Singapore embarked on resuming activities safely with a three-phased approach, ${ }^{8}$ the average number of participants in the open groups per week declined from 11 (during circuit breaker and phase 1 reopening) to 6 (phase 2). Through informal feedback, participants expressed no strong preference for virtual or face-to-face groups. However, several expressed that while virtual groups were preferred due to the reduction in travel time, participation during regular working hours was difficult once they had returned to their workplaces. This may partly explain the drop in participant numbers in phase 2 .

From our experience and feedback from patients, online groups can add considerable value to the care of our patients, and have the potential to operate in tandem with face-to-face groups in the future. The framework developed for online groups during circuit breaker will be a crucial part of our contingency plan for the future, on top of providing for patients who require individual support.

\section{The new norm}

At the time of writing, local transmission appears to be stabilising in Singapore. However, MDU is still unable to function as before in view of safety measures that are kept in place. A closely knit team that used to bond over breakfast meetings and outside working hours, it had to quickly adapt to virtual meetings and increased safe distancing. Nonetheless, there was a greater emphasis on teamwork, collaboration, problem-solving and selfcare during this period. Continuous learning and support were enabled virtually by ongoing clinical supervision, academic sessions, process groups and informal chat groups. These have contributed significantly to maintaining social connectedness and boosting staff morale, building the team's resilience to facing future challenges.

\section{Acknowledgements}

The authors thank colleagues Candice Sarah Ling Poh Hean, Chan Jiemin Priscilla, Chen Birong, Jessica Chan Boh Sze, Lim Wee Onn, Ng Choon Ming Louisa, Tang Wei Li Jonathan, Teresa Thia Hwei Ming, Vera Chua Ting Ru, and our nursing team at Mood Disorders Unit for sharing their professional views and experiences.

\section{REFERENCES}

1. Ho CS, Chee CY, Ho RC. Mental health strategies to combat the psychological impact of COVID-19 beyond paranoia and panic. Ann Acad Med Singap 2020;49:155-60.

2. Goh SSN, Chia MYC. Anxiety and morale in front-line healthcare workers during the coronavirus disease 2019 (COVID-19) outbreak at the national screening centre in Singapore. Ann Acad Med Singap 2020;49:259-62.

3. Hsu LY, Chia PY, Lim JF. The novel coronavirus (SARS-CoV-2) epidemic. Ann Acad Med Singap 2020;49:105-7.

4. Ministry of Health Singapore. Being prepared for a pandemic 2020. Available at: https://www.moh.gov.sg/diseases-updates/being-preparedfor-a-pandemic. Accessed on 1 July 2020.

5. Poremski D, Subner SH, Lam GFK, et al. Effective infection prevention and control strategies in a large, accredited, psychiatric facility in Singapore. Infect Control Hosp Epidemiol 2020;41:1238-40.

6. Ministry of Health Singapore. Circuit breaker to minimise further spread of Covid-19, 3 April 2020. Available at: https://www.moh.gov. sg/news-highlights/details/circuit-breaker-to-minimise-further-spreadof-covid-19. Accessed on 1 July 2020.

7. Wong CS, Mok YM, Wong FPY, et al. Adapting a mood disorders group therapy programme for the screen in the time of COVID-19. Singapore Med J 2020 (in press).

8. Ministry of Health Singapore. End of circuit breaker, phased approach to resuming activities safely, 19 May 2020. Available at: https://www.moh.gov.sg/news-highlights/details/end-of-circuitbreaker-phased-approach-to-resuming-activities-safely. Accessed on 11 August 2020. 